\title{
Evolution of Parallel Spindles Like genes in plants and highlight of unique domain architecture ${ }^{\#}$
}

\author{
Riccardo Aiese Cigliano ${ }^{1}$, Walter Sanseverino ${ }^{2}$, Gaetana Cremona ${ }^{1}$, Federica M Consiglio ${ }^{1}$ and Clara Conicella ${ }^{{ }^{*}}$
}

\begin{abstract}
Background: Polyploidy has long been recognized as playing an important role in plant evolution. In flowering plants, the major route of polyploidization is suggested to be sexual through gametes with somatic chromosome number (2n). Parallel Spindlel gene in Arabidopsis thaliana (AtPS1) was recently demonstrated to control spindle orientation in the 2 nd division of meiosis and, when mutated, to induce $2 n$ pollen. Interestingly, AtPS1 encodes a protein with a FHA domain and PINc domain putatively involved in RNA decay (i.e. Nonsense Mediated mRNA Decay). In potato, $2 n$ pollen depending on parallel spindles was described long time ago but the responsible gene has never been isolated. The knowledge derived from AtPS1 as well as the availability of genome sequences makes it possible to isolate potato PSLike (PSL) and to highlight the evolution of PSL family in plants.

Results: Our work leading to the first characterization of PSLs in potato showed a greater PSL complexity in this species respect to Arabidopsis thaliana. Indeed, a genomic PSL locus and seven cDNAs affected by alternative splicing have been cloned. In addition, the occurrence of at least two other PSL loci in potato was suggested by the sequence comparison of alternatively spliced transcripts.

Phylogenetic analysis on 20 Viridaeplantae showed the wide distribution of PSLs throughout the species and the occurrence of multiple copies only in potato and soybean.

The analysis of PSL ${ }^{\text {FHA }}$ and PSL ${ }^{\text {PINC }}$ domains evidenced that, in terms of secondary structure, a major degree of variability occurred in PINC domain respect to FHA. In terms of specific active sites, both domains showed diversification among plant species that could be related to a functional diversification among PSL genes. In addition, some specific active sites were strongly conserved among plants as supported by sequence alignment and by evidence of negative selection evaluated as difference between non-synonymous and synonymous mutations.

Conclusions: In this study, we highlight the existence of PSLs throughout Viridaeplantae, from mosses to higher plants. We provide evidence that PSLs occur mostly as singleton in the analyzed genomes except in soybean and potato both characterized by a recent whole genome duplication event. In potato, we suggest the candidate PSL gene having a role in $2 n$ pollen that should be deeply investigated.

We provide useful insight into evolutionary conservation of FHA and PINC domains throughout plant PSLs which suggest a fundamental role of these domains for PSL function.
\end{abstract}

\section{Background}

Polyploidy represents the occurrence of more than two complete sets of chromosomes in an organism and has long been recognized as playing an especially important role in plant evolution [1]. In flowering plants, polyploidy extent has been largely underestimated in terms

\footnotetext{
* Correspondence: conicell@unina.it

${ }^{1}$ CNR - National Research Council of Italy, Institute of Plant Genetics,

Research Division Portici, Via Università 133, 80055 Portici. Italy

Full list of author information is available at the end of the article
}

of its commonality. Indeed, major recent advances in genomic analysis has revealed that almost all angiosperms have experienced at least one round of whole genome duplication during their evolution.

The wide spreading of polyploidy throughout the angiosperms can be related to their highly plastic genome structure, as inferred from their tolerance to changes in chromosome number, genome size and epigenome [2]. Although information with regard to the modes of polyploidization is limited, the major route of
C Biomed Central

(c) 2011 Cigliano et al; licensee BioMed Central Ltd. This is an Open Access article distributed under the terms of the Creative Commons Attribution License (http://creativecommons.org/licenses/by/2.0), which permits unrestricted use, distribution, and reproduction in any medium, provided the original work is properly cited. 
polyploidization seems to be sexual through the functioning of gametes with somatic chromosome number ( $2 n$ gametes) [3]. Indeed, sexual polyploidization as compared to asexual would explain better the success of polyploid species in terms of higher fitness and more genetic flexibility. The control of $2 n$ gamete formation has been generally attributed to the action of single recessive genes. These genes exhibit incomplete penetrance and variable expression that is significantly influenced by genetic, environmental and developmental factors [4]. The molecular mechanisms leading to $2 n$ gametes have only recently begun to be uncovered $[5,6]$. In particular, d'Erfurth and colleagues [7] isolated and characterized Parallel Spindle1 gene in Arabidopsis thaliana (AtPS1) that controls diploid pollen formation through spindle orientation in the second division of meiosis. The occurrence of parallel spindles at meiosis II is a frequently found mechanism for $2 n$ pollen formation that was described in potato many decades ago $[8,9]$. In potato, $p s$ mutants have been used for breeding purposes in order to introgress beneficial traits from diploid $(2 n=2 x=24)$ relatives into cultivated strains [10]. However, the gene $p s$ leading to $2 n$ pollen via parallel spindles was not isolated, so far.

Interestingly, AtPS1 is a protein which contains contemporarily a ForkHead Associated domain (FHA), and a C-terminal PilT N-terminus domain (PINc). So far, the FHA domain has been found in more than 5600 different proteins from prokaryotes to higher eukaryotes involved in several processes including cell cycle control, DNA repair, protein degradation, transcription and premRNA splicing [11]. FHA domain was shown to recognize phosphothreonine-containing epitopes [12]. PINc domain has been found in more than 3600 proteins in all life kingdoms. PINc domain has RNA nuclease activity [13]. In eukaryotes, PINc-containing proteins, such as human SMG6 and SMG5, were linked to NonsenseMediated mRNA Decay (NMD), that recognizes and rapidly degrades $m \bar{R} N A s$ containing Premature translation Termination Codons (PTCs).

In this study, a sequence-homology-based strategy was carried out to isolate PS gene from a diploid potato. Through this approach, a genomic locus PS-Like (PSL) and seven cDNAs affected by alternative splicing have been cloned. The occurrence of at least two other PSL loci is suggested in potato. In order to shed light on the evolution and function of PSL genes in plants, a phylogenetic analysis of PSL genes was performed and FHA/ PINc domains were compared. As far as we know, this is the first report about the isolation and characterization of PSL in a crop. We also demonstrate the conservation of this gene family throughout plants.

\section{Results}

\section{Cloning and characterization of PSL genes in potato}

Using the predicted protein sequence of AtPS1, two ESTs were identified on DFCI Potato Gene Index (http://compbio.dfci.harvard.edu/tgi) named TC194262 and DN590600 corresponding to FHA and PINc domains, respectively. The primers designed on the above mentioned ESTs allowed to isolate in diploid potato a $3 \mathrm{Kbp}$ genomic clone (PSL1) lacking UTR regions. In order to complete genomic sequence of PSL1, by querying Potato Genome Sequencing Consortium Database (http://www.potatogenome.net/index. php) and SOL Genomic Network (http://solgenomics. net) we retrieved a $3 \mathrm{Kbp}$ region of $S$. phureja v3 scaffold PGSC0003DMS000001829 (phuPSL) and a tomato BAC clone AC211085.1 (SlPSL1) sharing 97\% and 93\% of sequence identity with PSL1, respectively. Primers designed on the tomato sequence were used to isolate a potato $5.3 \mathrm{Kbp} P S L 1$ genomic region spanning $2 \mathrm{Kbp}$ upstream of the start codon to about 200 bp downstream of the stop codon [GenBank:HQ418834]. In silico gene prediction showed a structure of PSL1 composed by six exons and five introns (Figure 1A) and a $2.4 \mathrm{Kbp}$ hypothetical PSL1 cDNA (PSL1_pred) with a GC content of $42 \%$ encoding a $92.5 \mathrm{kDa}$ protein of 823 aminoacids (pPSL1_pred).

Given the meiotic function of AtPS1, potato PSL1 cDNA was isolated from pre-bolting buds. Seven different cDNAs ranging from 2.3 to $2.7 \mathrm{Kbp}$ were isolated including PSL1a of $2.4 \mathrm{Kbp}$ corresponding to PSL1_pred. The sequence alignment between cDNAs and the genomic PSL1 indicated the presence of different groups of related PSL sequences encoded by more than one locus (Figure 1A). In order to investigate the relationship between the different PSL cDNAs, we conducted a phylogenetic analysis that suggested the existence of three different loci named PSL1, PSL2 and PSL3 (Figure 1B). On the basis of sequence similarity, we assigned PSL1a, PSL1b and PSL1c cDNAs [GenBank: HQ418835, GenBank:HQ418836 and GenBank: HQ418837] to genomic PSL1, PSL3a, PSL3b and PSL3c cDNAs [GenBank:HQ418839, GenBank:HQ418840 and GenBank:HQ418841] to PSL3 and the last cDNA to PSL2 [GenBank:HQ418838]. Moreover, the distance measured as the number of different nucleotides among the seven cDNAs showed that PSL2 was more similar to PSL3 than to PSL1 (Figure 1C). The nucleotide comparison between the predicted phuPSL cDNA and PSL1a, PSL2 and PSL3c cDNAs showing a similarity of $98.5 \%$, $99.1 \%$ and $99.6 \%$, respectively, suggested that phuPSL locus corresponds to PSL3 being the differences explained by the different genetic background. 


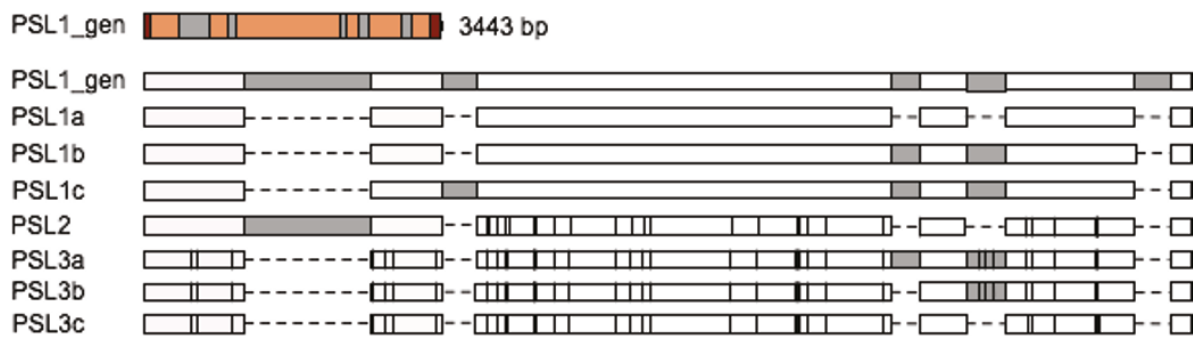

PSL3C

A

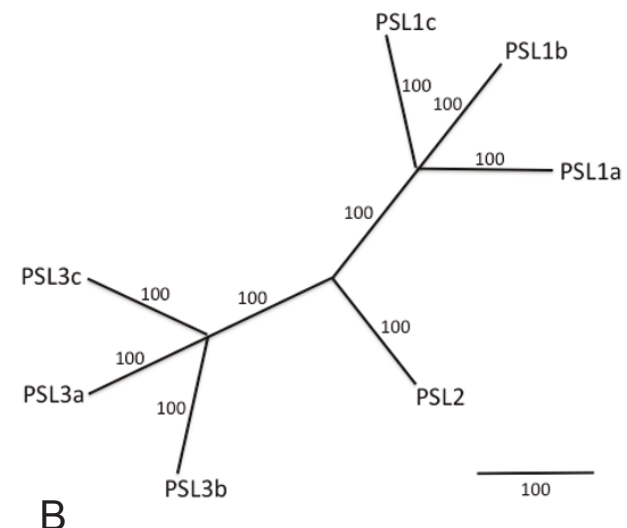

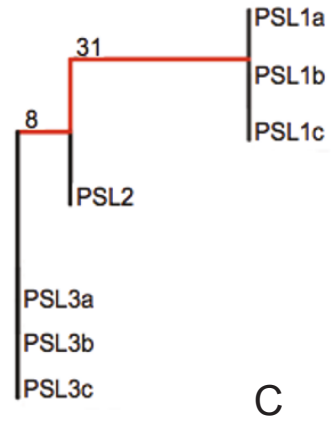

C

Figure 1 Potato PSL gene family. (A) PSL1 gene structure (PSL1_gen) with UTRs reported in red, exons in orange and introns in grey is showed together with the schematic alignment of the cloned CDNAs and PSL1_gen sequences, with exons in white, introns in grey and nucleotide differences respect to PSL1_gen as vertical black lines. (B) Maximum Likelihood phylogenetic tree of PSL CDNAs is reported with bootstrap values for each node and branch lengths measured in bootstrap values. (C) The distance in terms of number of different nucleotides is reported for the cloned PSL CDNAs. (D) Schematic representation of PSL predicted proteins obtained after in silico translation of the corresponding CDNAs showing FHA and PINc domains as ovals and exagons, respectively.

Based on the sequences of cloned PSL cDNAs, PSL1b and PSL1c, PSL3a and PSL3b are alternative splicing forms of PSL1 and PSL3 since they retained complete or partial introns causing the formation of premature stop codons (PTCs) (Figure 1A). Moreover, the cloned PSL2 cDNA showed a PTC caused by the retention of the second intron. As a consequence, all the predicted PSL proteins, except pPSL1a and PPSL3c, had truncated or lacking PINc domain (Figure 1D).

In order to evaluate whether the alternative splicing $P S L$ variants were possible target of degradation through NMD we calculated the distance between PTCs and the successive exon-exon junction. Being this distance more than 50-55 nt according to Nagi and Maquat [14] we could consider PSL1b, PSL1c, PSL2, PSL3a and PSL3b as target of NMD.

\section{Phylogenetic analysis of PSL genes in sequenced Viridaeplantae}

In order to investigate the evolution of $P S L$ family, a phylogenetic analysis was performed by a search on
Interpro (http://www.ebi.ac.uk/interpro) for proteins with both FHA and PINc domains. Interestingly, these domains were contemporary present only in plants, except for the multidrug-efflux transporter NAEGRDRAFT_78193 from the amoeboid Naegleria gruberi.

PSL1a sequence was blasted against the Phytozome v6 database (http://www.phytozome.net) that contains the genomic sequences of 22 organisms and against the SOL Genomics Network containing the tomato genome assembly. Afterwards, 25 sequences of predicted PSL proteins were collected from 19 different organisms (Additional File 1), since Physcomitrella patens, Ricinus communis, Volvox carteri, Zea mays and Chlamydomonas rehinardtii seemingly lack PSL genes. The sequences were then aligned and the Maximum-Likelihood phylogenetic tree is shown in Figure 2. The distribution of PSLs is in agreement with the known phylogenetic relationships between species among dicots and monocots. Moreover, one PSL locus was found in the analyzed plant species, except for Glycine max. Indeed, four different $P S L$ loci were identified in soybean and three of 


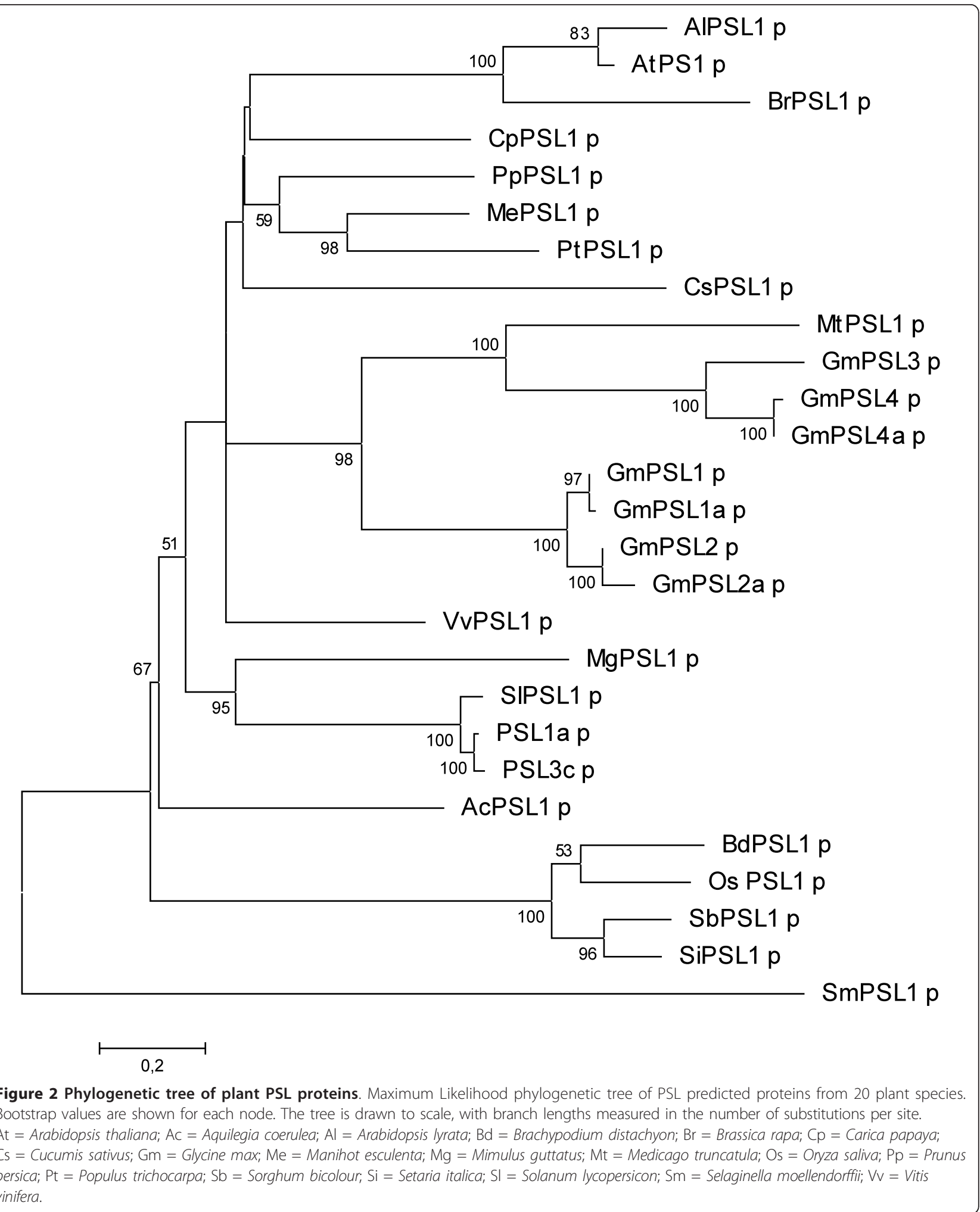




GmPSL1_gen
GmPSL1
GmPSL1a
GmPSL2_gen
GmPSL2
GmPSL2a
GmPSL3_gen
GmPSL3
GmPSL4_gen
GmPSL4
GmPSL4a
Figure 3 Alternative splicing of GmPSL genes. GmPSL1_gen,
GmPSL2_gen, GmPSL3_gen, GmPSL4_gen gene structures are
reported with UTRs in red, exons in orange and introns in grey. The
mRNAs are reported with UTRs in red, exons in orange and spliced
introns as interrupted lines underneath each gene structure. Arrow
indicates the lack of complete sequence.

them encode alternative transcripts. Differently from potato, the alternative transcripts of soybean retain PINc domain being the splicing sites located at the 3'-end of mRNA (Figure 3).

\section{Analysis of FHA and PINc secondary structure and active sites in PSL proteins}

In order to assess the conservation degree of PSLs, we predicted and compared the secondary structure of FHA and PINc domains using the SMART (http://smart. embl-heidelberg.de) and PSIPRED (http://bioinf.cs.ucl.ac. $\mathrm{uk} /$ psipred) tools. It is reported that FHA domain is 80100 aminoacid (aa) long folded into a 11-stranded beta sandwich, which sometimes contains small helical insertions between the loops connecting the strands. However, in silico analysis of FHA displays only 8 betastrands (b-strands) out of 11 including the residues involved in phosphopeptide recognition and stabilisation of domain architecture [15]. Using the above mentioned tools on yeast RAD53p [NCBI:6325104], a well characterized FHA containing protein [12], the identified FHA region was 52 aa covering 6 beta-strands (data not shown). As shown in Figure 4, the length of the predicted FHA region in our dataset was 53 aa except for Brassica rapa (51 aa) and for Glycine max PSL2 (68 aa). While the majority of FHA domains showed 6 betastrands, Brassica rapa FHA was predicted to be composed of 4 consecutive beta-strands followed by an alpha helical region. The group of monocots, Brachypodium distachyon, Oryza sativa and Sorghum bicolor, as well as dicot Glycine max (GmPSL1) showed a helical insertion between the 2nd and the 3rd beta-strand. In other species this helical insertion was predicted but at a low confidence value as estimated by PSIPRED.
Afterward, we compared the active sites in yRAD53p with those present in $\mathrm{PSL}^{\mathrm{FHA}}$ domains through the protein alignment showed in Figure 5. It can be observed that glycine- 5 , arginine- 6 , serine- 21 and histidine- 24 in FHA domain are perfectly conserved. The arginine-19 seems to be absent in all plant sequences. In the analyzed species, asparagine- 60 showed a substitution with histidine, characterized by a different polarity, except for soybean PSL4 glutamine-60 and Brassica rapa that seems to lack this site. Asparagine-66 is mostly substituted with the similar polar serine. Instead, AlPSL1, AtPSL1, GmPSL3 and GmPSL4 exhibited arginine-66 with different polarity while BrPSL1 glutammine-66 and MtPSL1 cysteine- 66 both showing similar polarity of asparagine.

The analysis of PINc domain in our dataset started with the prediction of its secondary structure in human SMG6 (hSMG6) [UniProt:Q86US8]. It is reported that hSMG6 $^{\text {PINc }}$ (hSMG6) is 182 aa folded into a 5-stranded parallel beta-sheets that is highly twisted and alphahelices arranged on both sides of each beta-sheet for a total of 6. Three aspartate residues are essential for PINc activity in hSMG6, while a threonine or a serine in the sequence (T/S)XD might be involved in catalytic role on the basis of similarity with other PINc domains $[16,17]$. SMART predicted a PINc domain of 152 aa lacking the first and the last alpha-helices while PSIPRED predicted 4 beta-sheets and 4 alpha helices in hSMG6 (data not shown). In our dataset the PINc domain ranged from a minimum of 123 aa in Cucumis sativus to a maximum of 162 aa in Brachypodium distachyon (Figure 6). In our prediction, the number of beta-sheets ranged from 3 in GmPSL3 and CsPSL1 to 9 in CPPSL1. The number of predicted alpha-helices ranged from 3 in CsPSL1, OsPSL1 and PSL3c to 7 in BdPSL1 and CPPSL1. Afterward, we compared the active residues of PINc in PSL proteins. The alignment between PINc domain of PSL proteins and hSMG6 is shown in Figure 7 (refer to Additional file 2: Alignment of PSL ${ }^{\text {PINc }}$ and hSMG6 ${ }^{\text {PINc }}$ residues to look at raw alignment). BrPSL1, SmPSL1 and PSL3c show the three expected aspartate residues at positions 6, 194 and 233 of the alignment. SiPSL1 and SbPSL1 have a substitution of two aspartate residues with asparagine-6, glutammate-194. When a substitution occurs, the aspartate-194 is mostly replaced with glutammate-194 except for CpPSL1 showing a different polarity residue (lysine-194) and MgPSL1 showing an alyphatic residue (alanine-194). The aspartate-233 is widely conserved except in BdPSL1, OsPSL1 and SiPSL1 where it is substituted with a serine-233. Most of the PSL proteins show a catalytic serine-231 in the described pattern SXD (where X can be $\mathrm{D}, \mathrm{N}, \mathrm{E}$ or $\mathrm{S}$ in this study) instead of threonine231 in hSMG6, PtPSL1, SiPSL1, SmPSL1 and VvPSL1. 


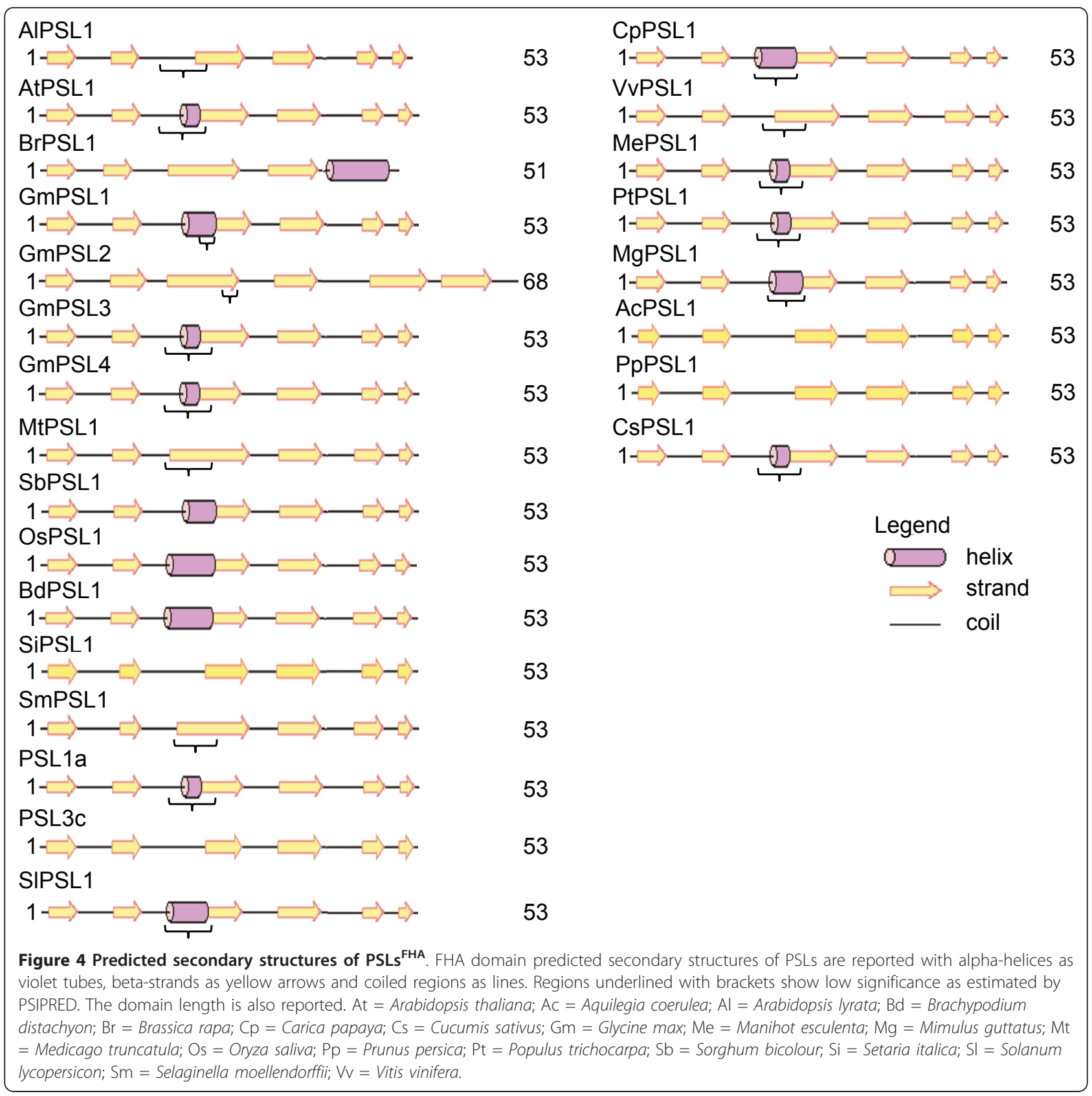

GmPSL3 and SbPSL1 are the only proteins lacking the C-terminal extremity of PINc interrupting at leucine203 and lysine-227 respectively thus missing the catalytic threonine/serine-231 and the aspartate- 233 .

\section{Selective pressure among amino acid sites in the PSL family}

In order to test for presence of positive selection at individual amino acid codons, the site specific models implemented in DataMonkey 2010 webserver (http:// www.datamonkey.org) [18] were used. The Integrative Selection Analysis of FEL, SLAC and FER alghoritms evidenced no significant positive selected sites $(\mathrm{dN}-\mathrm{dS}>$ $0)$. Conversely, 217 significant negative selected sites $(\mathrm{dN}-\mathrm{dS}<0)$ were identified. The region between FHA to PINc domains included few negative selected sites which were mostly located near the functional domains. The codons encoding the highly conserved active sites glycine-5, arginine-6, serine-21 and histidine-24 in FHA and aspartate-6, serine/threonine-231 and aspartate-233 in PINc were also subjected to negative selection (Figure 8). These observations support the results obtained through sequence alignment and evidence an occurrence of negative pressure upon non-synonymous 


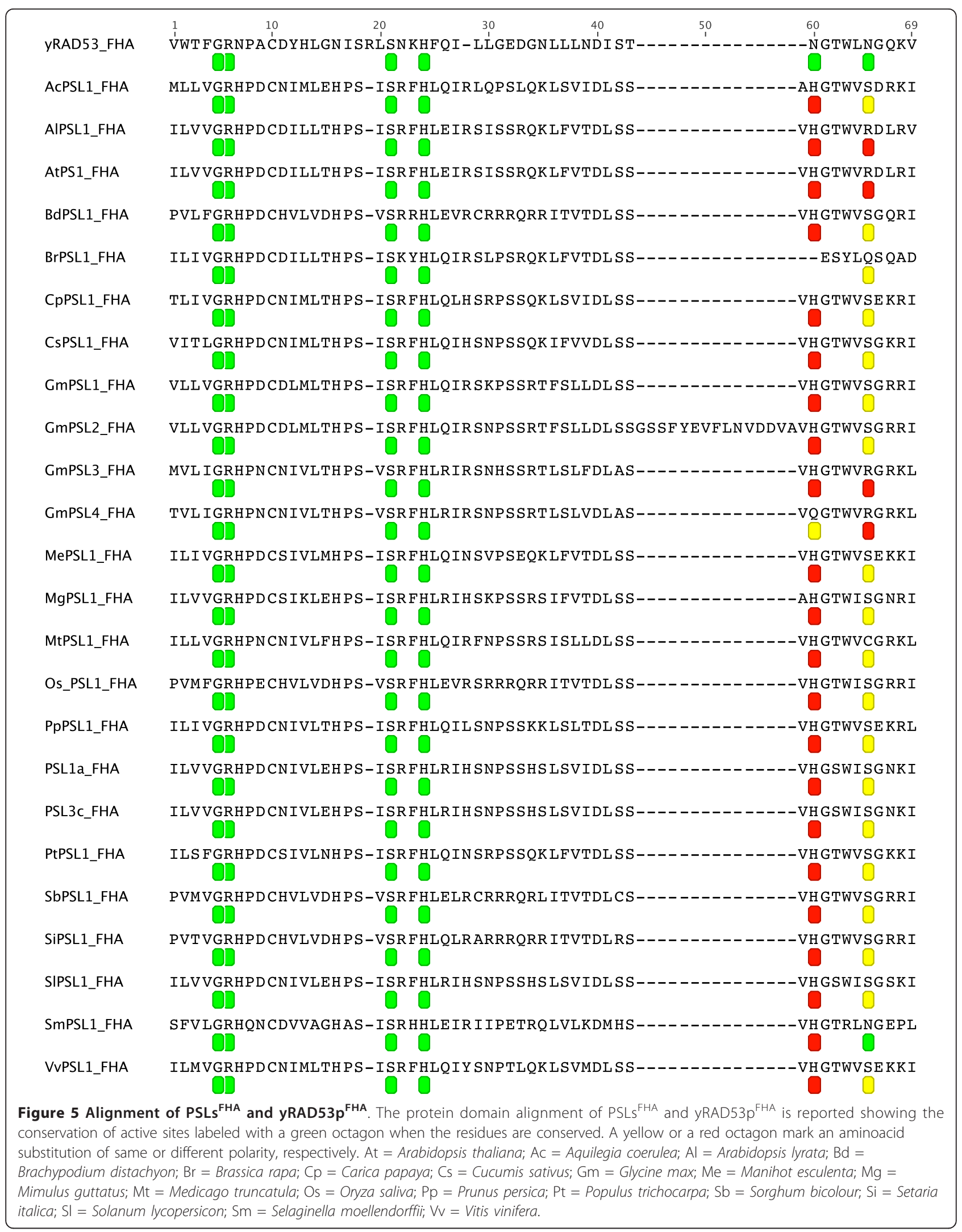




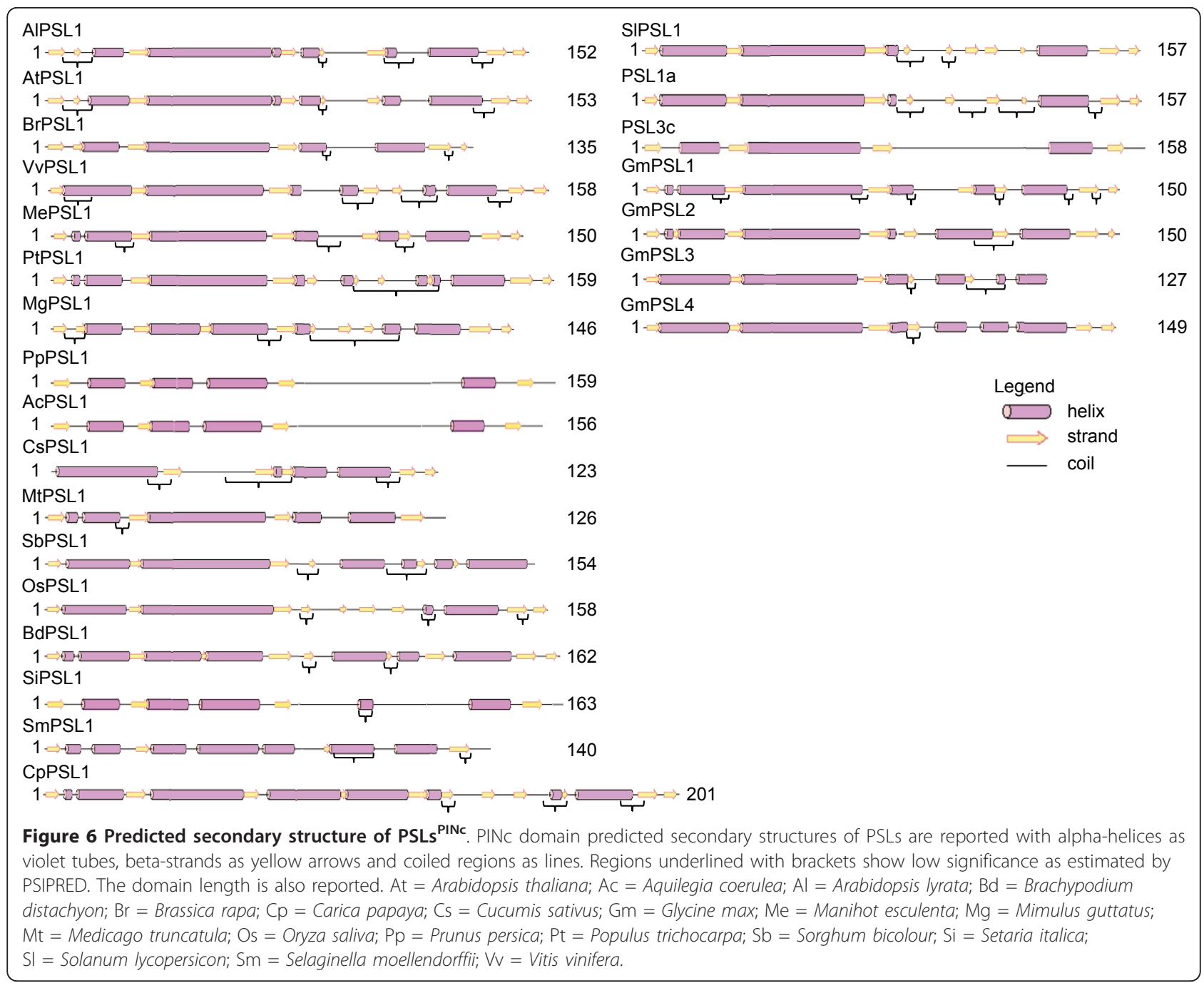

mutations in PSL genes. In particular, the regions next to the functional domains and the codons for active sites were affected.

\section{Discussion}

In this work, we isolated PSL genes in a diploid potato $(2 n=2 x=24)$ and through in silico analysis we identified PSL in other plant species. The function of PSL in plants can be inferred from Arabidopsis study on AtPS1 gene that appears responsible for the spindle orientation at meiosis II playing a regulatory function, likely via RNA decay [7].

In all the analysed species, except soybean and potato, PSL loci appear as singleton behaving as resistant to duplication. It is known that angiosperms are mostly paleopolyploids [19], many having survived multiple duplication events [20]. Analysis of genome sequences shows that some genes duplicate and persist as multiple copies after whole genome duplication (WGD) while other genes are iteratively returned to singleton status. Moreover, it seems that the singleton status is consistently restored for some functional gene groups like those involved in DNA repair or signal transduction [21]. The evolutive history of potato and soybean could explain the expansion of PSL genes occurring in these species that have experienced recent WGD. In cultivated potato $(2 n=4 x=48)$, WGD is reported to have occurred about 10 million of years ago (mya) after the divergence from Solanum lycopersicum [22]. In soybean $(2 n=2 x=40)$, which seems to be an ancient allopolyploid on the basis of two different centromeric repeat classes [23], two rounds of WGD happened the latter aging 10-15 mya [24,25]. In addition, the location of the four soybean PSLs on different chromosomes reinforce their origin from WGD rather than tandem duplications.

The evident feature of PSL proteins is the contemporary presence of FHA and PINc domains. FHA is reported as a phosphothreonine ( $\mathrm{pT}$ ) binding domain 


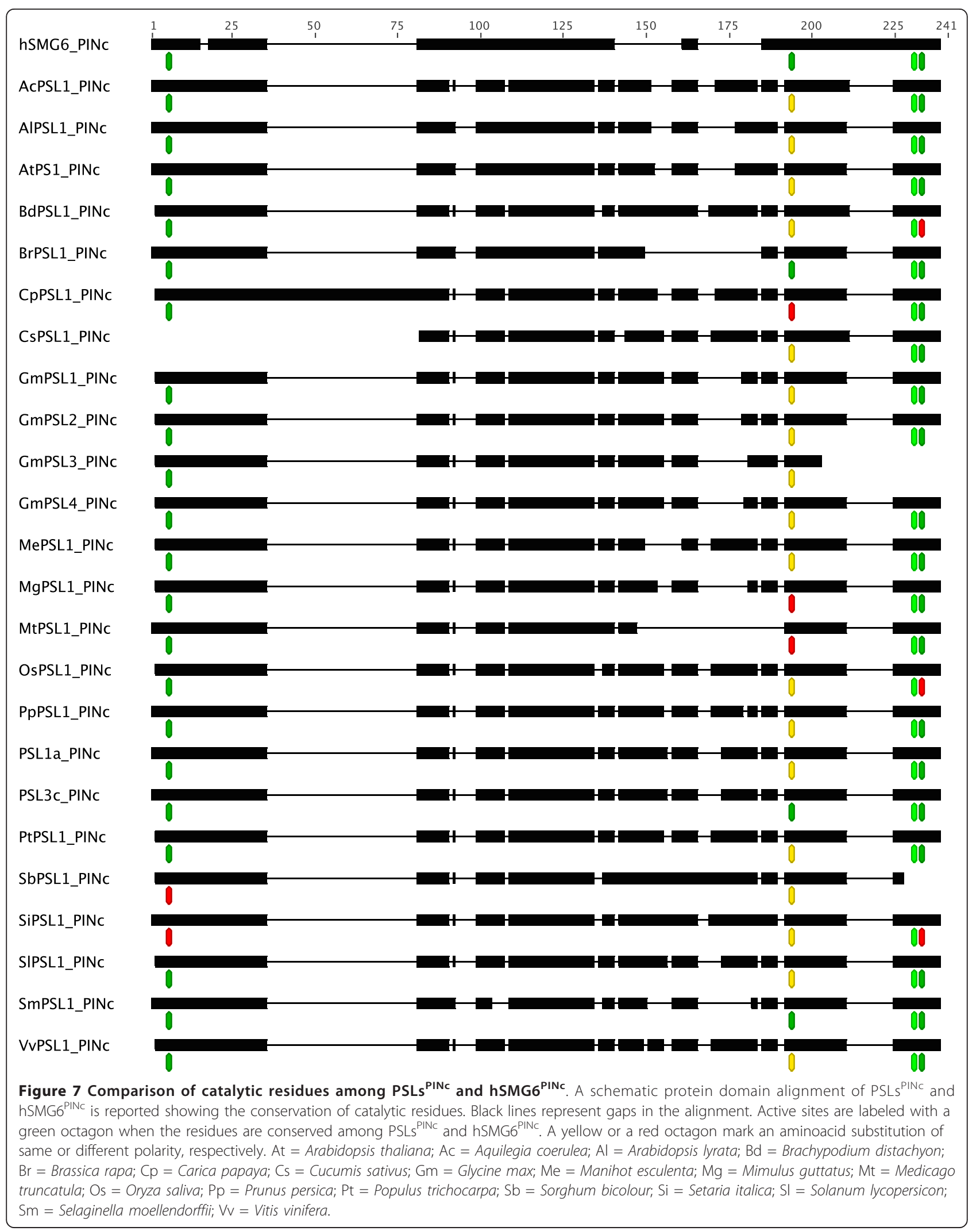




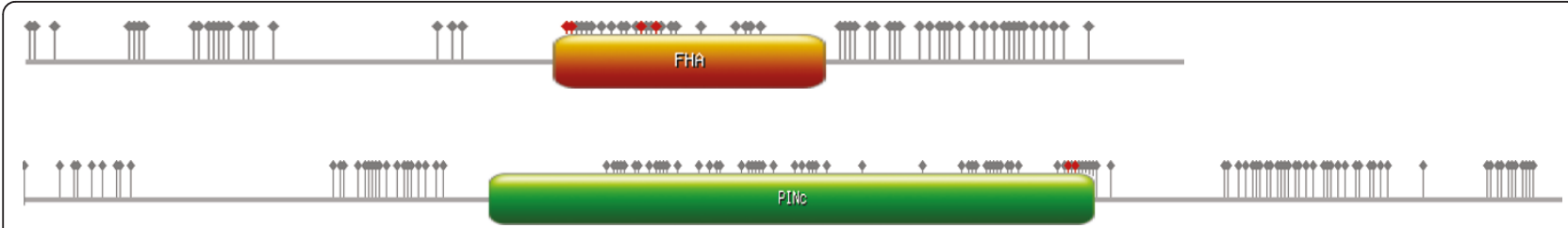

Figure 8 Distribution of negatively selected codons in $\mathrm{PSL}^{\mathrm{FHA}}$ and $\mathrm{PSL}^{\mathrm{PINc}}$. A schematic representation of FHA and PINc domains is reported showing negatively selected sites as grey diamonds. Red diamonds mark active sites subjected to negative selection.

showing a 11 beta-sandwich secondary structure, also containing small helical insertions between the betastrands [15]. The FHA active sites are usually located in the loops connecting $\mathrm{b} 3 / \mathrm{b} 4, \mathrm{~b} 4 / \mathrm{b} 5$ and $\mathrm{b} 6 / \mathrm{b} 7$ strands. RAD53 $\mathrm{p}^{\mathrm{FHA}}$ arginine-70, serine- 85 and asparagine- 107 (corresponding to arginine-6, serine-21 and asparagine60 in Figure 5) are involved in the interaction with the phosphopeptide backbone. Arginine-83 (corresponding to arginine-19 in Figure 5) is the most important aminoacid for FHA binding specificity. Indeed, its conversion to glycine shifts the binding from pTXXD to pTXXI peptides in RAD53p ${ }^{\mathrm{FHA}}$ interaction experiments using surface plasmon resonance [15]. Glycine-69 and histidine-88 (corresponding to glycine- 5 and histidine24 in Figure 5) stabilize the architecture of the binding site. The remaining conserved residue, asparagine- 112 (corresponding to asparagine-65 in Figure 5), is remote from the peptide binding site and serves to tether the beta turn between b7/8 to b10 [12,15]. The comparison of predicted secondary structure in our dataset showed a wide conservation among plant species regarding the number and the position of the beta-sheets and the presence of an helical insertion between the second and the third beta-sheet as a characteristic feature of monocot PSL proteins. The comparison of active sites showed that two PSL ${ }^{\mathrm{FHA}}$ residues involved in pT binding as well those involved in stabilisation of the architecture of the binding site are fully conserved except for asparagine- 65 that is replaced with a different polarity residue (arginine) in AlPSL1, AtPS1, GmPSL3 and GmPSL4 with unknown possible effect on domain architecture. Moreover, as compared to RAD53 $\mathrm{p}^{\mathrm{FHA}}$, a conserved substitution in plants is a histidine instead of asparagine- 60 , known to be important for the selectivity of binding of phosphothreonine upon phosphoserine. The different polarity between these two residues might suggest a functional diversification of this active site. However, the consequences of this substitution in $\mathrm{PSL}^{\mathrm{FHA}}$ domain in terms of ligand binding cannot be easily predicted and should be assessed by protein:protein interaction analysis. It is also intriguing the lack of arginine-19 in plants raising the question of binding selectivity for plant $\mathrm{PSL}^{\mathrm{FHA}}$.
As regards PINc domain, we made reference to human SMG6 ${ }^{\text {PINc }}$ which has RNAse activity and it is composed of alternating beta-sheets and alpha-helices. It is reported that hSMG6 is involved in NMD together with hSMG5 and hSMG7 [25]. In Arabidopsis, AtSMG7 was proved to be involved in NMD and to be required for meiotic spindle organization in meiosis II [26]. As reported by Glavan and colleagues [17], hSMG6 and other PINc domains show three conserved aspartic residues at positions 1251, 1353 and 1392 (corresponding to aspartate-6, -194 and -233 in Additional File 2) involved in $\mathrm{Mg}^{2+}$ binding. Threonine or serine embedded in the motif $(\mathrm{T} / \mathrm{S}) \mathrm{XD}$ is proposed to be the catalytic site on the basis of sequence alignment of PIN domains and it is located at residue-1390 (corresponding to threonine-231 in Figure 7) in hSMG6 ${ }^{\mathrm{PINc}}$.

Our results showed differences of secondary structures in PSL ${ }^{\text {PINc }}$ domains even between phylogenetically close organisms. Moreover, the typical alternation between beta-sheets and alpha-helices does not seem to be respected. In spite of these differences, the active sites showed a wide conservation among plants. The aspartate- 6 is conserved in all proteins except for SbPSL1 and SiPSL1 where an asparagine is present. This substitution maybe occurred after the divergence of BEP from PACCAD clades among Poaceae given its absence in Oryza sativa, Brachypodium distachyon and Selaginella moellendorffii. The residue-194 showed the major degree of variation among the species mostly characterized by glutamate instead of aspartate. This substitution is not related to phylogenesis, being glutamate present in both monocots and dicots while aspartate-194 is shared by the Brassicaceae BrPSL1, the Selaginellaceae SmPSL1 and the Solanaceae PSL3c. In Carica papaya, Mimulus guttatus and Medicago truncatula the change involved aminoacids with a different polarity such as lysine, alanine, and asparagine. The aspartate- 233 is widely conserved among PSL proteins, except those of Poaceae OsPSL1, SiPSL1 and BdPSL1 showing a serine-233, likely a substitution occurred after the separation of monocots from dicots. GmPSL3 and SbPSL1 lack this residue due to PINc domain truncation at leucine-203 and lysine-227, respectively. As compared to hSMG6, 
the majority of plants showed a serine instead of threonine-231 that is present only in PtPSL1, SiPSL1, SmPSL1 and VvPSL1. This substitution should not compromise the PINc activity since serine and threonine are the most represented residues at this position in PINc domains of different organisms [16]. Based on the evidence that the three aspartate residues of PINc domain are crucial for RNase activity, we can argue that PSLs lacking one of these residues or showing aminoacid with different polarity (BdPSL1, CpPSL1, CsPSL1, GmPSL3, MgPSL1, MtPSL1, SbPSL1, SiPSL1, OsPSL1) have no enzymatic activity. However, we cannot exclude that they are partners of other proteins retaining RNAse activity. Indeed, in human, it is known that hSMG5 lacking two aspartate residues respect to hSMG6 has no enzymatic activity but the interaction between hSMG5 and hSMG7 led to a functional nuclease activity of SMG7-SMG5 complex [17].

Functional analysis of Arabidopsis PS1 reinforced the evidence that the defects in meiotic spindle orientation in meiosis II led to the formation of diplopollen. Among the analysed species, Manihot esculenta $(2 n=2 x=36)$ was reported to produce $2 n$ pollen but the cytological mechanisms underlying its formation were not deeply investigated [27]. Since the predicted MePSL protein has FHA and PINc active sites similar to those of other species, it is likely that the mechanism leading to $2 n$ pollen in Manihot esculenta does not involve parallel spindles.

In the potato genotype analysed in this study, neither spindle defects nor $2 n$ pollen have been reported [28]. In this genotype, we identified three PSL loci and seven transcripts. Based on AtPS1 characterized by FHA and PINc domains we can suspect that PSL1a and PSL3c, carrying both domains, are functional proteins. In addition, it can be speculated that PSL1a that evidences the same PINc active residues of AtPS1 is the strongest candidate for the regulation of spindle orientation in meiosis II. The landscape of alternative splicing in potato PSL is not surprising since it has been already observed for genes involved in Arabidopsis meiosis. For instance, AtSPO11-1, involved in double strand breaks (DSBs) required for meiotic recombination, exhibits up to ten splicing forms showing PTCs [29]. As inferred for AtSPO11-1, PSLs are possible target of NMD that could act as a post-transcriptional regulatory pathway for the proper expression of PSL. Alternative splicing was observed also in Glycine max but the lack of PTCs exclude NMD regulation of PSL transcripts. Defining the ligands of FHA and PINc domains and proving PSL as a component in NMD are essential to link PSLs to plant evolution by polyploidization via $2 n$ gametes.

\section{Conclusions}

In this study, we show that PSLs are common genes across Viridaeplantae. We provide evidence that PSLs occur mostly as singleton in the analyzed genomes except in soybean and potato both characterized by a recent event of whole genome duplication. We provide useful insight into evolutionary preservation of FHA and PINc domains throughout plant PSL genes, suggesting a fundamental role of these domains for PSL function. FHA appeared to be highly conserved, while PINc secondary structure and specific active sites showed a less conserved landscape, suggesting a functional diversification among PSL genes.

\section{Methods}

Plant material

A previously described [30] diploid clone of potato (named T710) coming from hybridization between Solanum tuberosum haploid USW3304 $(2 n=2 x=24)$ and S. chacoense $(2 n=2 x=24)$ has been used to isolate PSL genes.

\section{Potato PSL cloning and sequence analysis}

Plant genomic DNA was isolated from leaves using the DNeasy Plant Mini Kit (QIAGEN http://www1.qiagen. com). Bacterial plasmid DNA was isolated using QIAprep Spin Miniprep Kit (QIAGEN). Total RNA from prebolting buds was extracted using the RNeasy Plant Mini Kit (QIAGEN) and then treated with DNase I (Invitrogen, http://www.invitrogen.com) to remove residual genomic DNA. Primer pairs for cloning designed with PRIMER3 (http://frodo.wi.mit.edu/cgi-bin/primer3/ primer3.cgi) are listed in Additional file 3: Primers used in this study.

PSL1 genomic fragment was amplified by PCR with primers PS_F1 and PS_R1 and full sequence with primers PS_F2 and PS_R2. The coding region of PSL transcripts was amplified by RT-PCR by using SuperScriptTM III Reverse Transcriptase (Invitrogen) with an oligo- $\mathrm{dT}_{12-18}$ (Invitrogen). The cDNA was subjected to PCR with PS_CF and PS_CR primers (Additional File 3). PCR products have been cloned into PCR2.1 with T/A Cloning kit (Invitrogen). Nucleotide sequencing was carried out by Eurofins MWG Operon sequencing service (Germany).

PSL1 gene structure and cDNA predictions were carried out using FGENESH online tool (http://linux1.softberry.com/berry.phtml?topic $=$ fgenesh $\&$ group $=$ pro grams\&subgroup=gfind) selecting Tomato as organism. In silico translation of cDNA sequences was carried out using the Expasy Translation tool (http://www.expasy. ch/tools/dna.html). 


\section{Identification of unannotated PSL genes}

The PSL protein sequences were identified and collected by TBLASTN [31] search against the Phytozome v6 database (http://www.phytozome.net/), SGN (SOL Genomic Network, http://sgn.cornell.edu/) and Potato Genome Sequencing Consortium (PGSC, http://potatogenomics.plantbiology.msu.edu/index.php?p=blast).

\section{Molecular Phylogenetic analysis by Maximum Likelihood method}

The PSL protein sequences were firstly aligned by MUSCLE [32] using the default settings of MEGA5 [33]. The best model for Maximum Likelihood phylogeny analysis was chosen testing all the available models in ProtTest version 2.4 [34] with slow optimization strategy and selecting the one with highest AICc value. The evolutionary history was then inferred by using the Maximum Likelihood method according to Jones et al. w/freq. model [35]. The bootstrap consensus tree was inferred from 100 replicates [36]. Initial tree(s) for the heuristic search were obtained automatically as following. When the number of common sites is $<100$ or less than one fourth of the total number of sites, the maximum parsimony method was used, otherwise BIONJ method with MCL distance matrix was used. A discrete Gamma distribution was used to model evolutionary rate differences among sites (4 categories, $+\mathrm{G}$, parameter $=1.3900)$. The rate variation model allowed for some sites to be evolutionarily invariable $([+\mathrm{I}]$, $9.0566 \%$ sites). All ambiguous positions were removed for each sequence pair. Evolutionary analyses were conducted in MEGA5.

\section{PSL domain analysis}

The locations of FHA and PINc domains within PSL genes were detected using SMART [37]. Secondary structure prediction was performed using domains from PSL sequences as input into the PSIPRED secondary structure prediction server [38]. The program MUSCLE [32] was used to do multiple sequence alignments of FHA and PINc domains in PSL proteins, yRAD53p [NCBI:6325104] and hSMG6 [UniProt: Q86US8]. Conservation of phosphothreonine-binding residues in FHA and of RNAse activity residues in PINc were determined by alignment with yRAD53p and hSMG6, respectively.

\section{Comparison of $\mathrm{dN}$-dS values between PSL sequences}

The coding sequences of PSLs were obtained from the databases reported in Additional File 1. The terminal codon was manually removed, then the codon alignment was performed by MUSCLE [32] using the default settings of MEGA5 [33] (Tamura et al., personal communication). To select the best substitution model we used JmodelTest 0.1.1 [39,40] with default settings. The GTR [41] model was selected as the best fitting as evidenced by AICc values.

The codon alignment was then uploaded on DataMonkey 2010 server [18,42] and dN-dS evaluated using GTR as substitution model, and SLAC (default settings except for Global $\mathrm{dN} / \mathrm{dS}$ value = estimated with $\mathrm{CI}$ ), FEL and REL [43] alghoritms. Codons subjected to evolutive pressure were identified with Integrative Selection Analysis selecting significance levels for SLAC $\mathrm{p}<0.1$, FEL $\mathrm{p}<0.1$ and REL Bayes Factor $<50$. Only codons with a significant $\mathrm{dN}-\mathrm{dS}$ value according to all the three methods were reported.

\section{Note}

\#Contribution n 358 from CNR - National Research Council of Italy, Institute of Plant Genetics, Research Division Portici.

\section{Additional material}

Additional file 1: PSL sequences used in this study. Description of sequence names, accession numbers, organisms and source databases used in this study.

Additional file 2: Alignment of PSL ${ }^{\mathrm{PINc}}$ and $\mathrm{hSMG} 6^{\mathrm{PINc}}$ residues. The protein domain alignment of PSLs ${ }^{\mathrm{PINC}}$ and $\mathrm{hSMG} 6^{\mathrm{PINC}}$ is reported showing the conservation of catalytic residues. Dotted lines represent gaps in the alignment. Active sites are labeled with a green octagon when the residues are conserved among PSLs ${ }^{\text {PINC }}$ and $h S M G 6^{\text {PINC }}$. A yellow or a red octagon mark an aminoacid substitution of same or different polarity, respectively.

Additional file 3: Primers used in this study. Primers used for the isolation of PSL genomic clone and CDNAS

\section{Acknowledgements}

We wish to thank Ms. Rosa Paparo for helpful technical assistance, Prof. Domenico Carputo and Dr. Raphael Mercier for useful comments on the manuscript. This work was partially funded by Galileo Grant from University Italy-France.

\section{Author details}

${ }^{1}$ CNR - National Research Council of Italy, Institute of Plant Genetics, Research Division Portici, Via Università 133, 80055 Portici. Italy. ${ }^{2}$ DISSPAPA, Dept. of Soil, Plant and Environmental Sciences, University of Naples "Federico II", Via Università 100, 80055 Portici. Italy.

\section{Authors' contributions}

RAC performed the molecular research and the protein domain analysis, participated in phylogenetic analysis, study design and drafted the manuscript. WS carried out the phylogenetic analysis and participated in bioinformatic analysis design. GC participated in molecular analysis. FMC performed the molecular analysis design and revised the manuscript. CC conceived of and coordinated the study, and revised the manuscript. All authors read and approved the final manuscript.

Received: 26 November 2010 Accepted: 24 March 2011

Published: 24 March 2011 


\section{References}

1. Tate JA, Soltis DE, Soltis PS: Polyploidy in Plants. In The Evolution of the Genome. Edited by: Gregory T. Elsevier Inc; 2005:371-414.

2. Leitch AR, Leitch IJ: Genomic plasticity and the diversity of polyploid plants. Science 2008, 320:481-483.

3. Bretagnolle F, Thompson J: Tansley Review No-78 - Gametes with the somatix chromosome-number - mechanisms of their formation and rolein the evolution of autopolyploid plants. New Phytol 1995, 129(1):1-22

4. Consiglio F, Carputo D, Frusciante L, Monti LM, Conicella C: Meiotic mutations and crop improvement. In Plant Breeding reviews. Volume 28 Edited by: Janick J. Purdue University, John Wiley 2007:163-203.

5. Mercier R, Vezon D, Bullier E, Motamayor J, Sellier A, Lefevre F, Pelletier G, Horlow C: SWITCH1 (SWI1): a novel protein required for the establishment of sister chromatid cohesion and for bivalent formation at meiosis. Gene Dev 2001, 15(14):1859-1871.

6. Ravi M, Marimuthu MPA, Siddiqi I: Gamete formation without meiosis in Arabidopsis. Nature 2008, 451:1121-1124.

7. d'Erfurth I, Jolivet S, Froger N, Catrice O, Novatchkova M, Simon M, Jenczewski E, Mercier R: Mutations in AtPS1 (Arabidopsis thaliana parallel spindle 1) lead to the production of diploid pollen grains. PLOS Genet 2008, 4:e1000274

8. Fukuda Y: Cytological studies on the development of pollen grains in different races of solanum. Bot Mag Tokyo 1927, 41:459-474.

9. Mok D, Peloquin J: The inheritance of three mechanisms of diplandroid ( $2 n$ pollen) formation in diploid potatoes. Heredity 1975, 35:1-8.

10. Peloquin SJ, Boiteux LS, Carputo D: Meiotic mutants in potato. Valuable variants. Genetics 1999, 153:1493-1499.

11. Li J, Lee Gl, Van Doren SR, Walker JC: The FHA domain mediates phosphoprotein interactions. J Cell Sci 2000, 113:4143-4149.

12. Durocher D, Jackson SP: The FHA domain. FEBS Lett 2002, 513:58-66.

13. Lareau LF, Brooks AN, Soergel DAW, Meng Q, Brenner SE: The coupling of alternative splicing and nonsense-mediated mRNA decay. Adv Exp Med Biol 2007, 623:190-211.

14. Nagy $E$, Maquat $L E:$ A rule for termination-codon position within introncontaining genes: when nonsense affects RNA abundance. Trends Biochem Sci 1998, 23(6):198-199.

15. Durocher D, Taylor IA, Sarbassova D, Haire LF, Westcott SL, Jackson SP, Smerdon SJ, Yaffe MB: The molecular basis of FHA domain: phosphopeptide binding specificity and implications for phosphodependent signaling mechanisms. Mol Cell 2000, 6(5):1169-1182.

16. Arcus VL, Bäckbro K, Roos A, Daniel EL, Baker EN: Distant structural homology leads to the functional characterization of an archaeal PIN domain as an exonuclease. J Biol Chem 2004, 279(16):16471-16478.

17. Glavan F, Behm-Ansmant I, Izaurralde E, Conti E: Structures of the PIN domains of SMG6 and SMG5 reveal a nuclease within the mRNA surveillance complex. The EMBO Journal 2006, 25(21):5117-5125.

18. Pond SLK, Frost SDW: Datamonkey: rapid detection of selective pressure on individual sites of codon alignments. Bioinformatics 2005, 21(10):2531-2533.

19. Bowers JE, Chapman BA, Rong J, Paterson AH: Unravelling angiosperm genome evolution by phylogenetic analysis of chromosomal duplication events. Nature 2003, 422(6930):433-438

20. Paterson AH, Bowers JE, Chapman BA: Ancient polyploidization predating divergence of the cereals, and its consequences for comparative genomics. Proc Natl Acad Sci USA 2004, 101(26):9903-9908.

21. Doyle JJ, Flagel LE, Paterson AH, Rapp RA, Soltis DE, Soltis PS, Wendel JF: Evolutionary genetics of genome merger and doubling in plants. Annu Rev Genet 2008, 42:443-461.

22. Fawcett JA, Maere S, Van de Peer Y: Plants with double genomes might have had a better chance to survive the Cretaceous-Tertiary extinction event. Proc Natl Acad Sci USA 2009, 106(14):5737-5742.

23. Gill N, Findley S, Walling JG, Hans C, Ma J, Doyle J, Stacey G, Jackson SA: Molecular and chromosomal evidence for allopolyploidy in soybean. Plant Physiol 2009, 151(3):1167-1174.

24. Shoemaker RC, Schlueter J, Doyle JJ: Paleopolyploidy and gene duplication in soybean and other legumes. Curr Opin Plant Biol 2006, 9(2):104-109.

25. Chang Y-F, Imam JS, Wilkinson MF: The nonsense-mediated decay RNA surveillance pathway. Annu Rev Biochem 2007, 76:51-74.
26. Riehs N, Akimcheva S, Puizina J, Bulankova P, Idol RA, Siroky J, Schleiffer A, Schweizer D, Shippen DE, Riha K: Arabidopsis SMG7 protein is required for exit from meiosis. J Cell Sci 2008, 121:2208-2216.

27. Ogburia M, Yabuya T, Adachi T: A cytogenetic study of bilateral sexual polyploidization in cassava (Manihot esculenta Crantz). Plant Breeding 2002, 121:278-280

28. Conicella C, Barone A, Giudice A, Frusciante L, Monti LM: Cytological evidences of SDR-FDR mixture in the formation of $2 \mathrm{n}$ eggs in a potato diploid clone. Theor App/ Genet 1991, 81(1):59-63.

29. Hartung F, Puchta H: Molecular characterisation of two paralogous SPO11 homologues in Arabidopsis thaliana. Nucleic Acids Res 2000, 28(7):1548-1554

30. Barone C, Frusciante : Selection of potato diploid hybrids for $2 n$ gamete production. Journal of Genetics and Breeding 1993, 47:313-318.

31. Altschul SF, Lipman DJ: Protein database searches for multiple alignments. Proc Natl Acad Sci USA 1990, 87(14):5509-5513.

32. Edgar RC: MUSCLE: multiple sequence alignment with high accuracy and high throughput. NAR 2004, 32(5):1792-1797.

33. Tamura K, Dudley J, Nei M, Kumar S: MEGA4: Molecular Evolutionary Genetics Analysis (MEGA) software version 4.0. Mol Biol Evol 2007, 24:1596-1599.

34. Abascal F, Zardoya R, Posada D: ProtTest: Selection of best-fit models of protein evolution. Bioinformatics 2005, 21:2104-2105.

35. Jones DT, Taylor WR, Thornton JM: The rapid generation of mutation data matrices from protein sequences. Comput Appl Biosci 1992, 8(3):275-282.

36. Felsenstein : Confidence limits on phylogenies: An approach using the bootstrap. Evolution 1985, 39:783-791.

37. Schultz J, Milpetz F, Bork P, Ponting CP: SMART, a simple modular architecture research tool: identification of signaling domains. Proc Natl Acad Sci USA 1998, 95(11):5857-5864.

38. McGuffin LJ, Bryson K, Jones DT: The PSIPRED protein structure prediction server. Bioinformatics 2000, 16(4):404-405.

39. Posada D: jModelTest: Phylogenetic Model Averaging. Mol Biol Evol

40. Guindon S, Gascuel O: A simple, fast and accurate method to estimate large phylogenies by maximum-likelihood. Syst Biol 2003, 52:696-704.

41. Tavaré S: Some Probabilistic and Statistical Problems in the Analysis of DNA Sequences. In Lectures on Mathematics in the Life Sciences Edited by: AMS 1986, 17:57-86.

42. Pond SLK, Muse SV: HyPhy: hypothesis testing using phylogenies. Bioinformatics 2005, 21(5):676-679.

43. Pond SLK, Frost SDW: Not So Different After All: A Comparison of Methods for Detecting Amino Acid Sites Under Selection. Mol Biol Evol 2005, 22(5):1208-1222.

doi:10.1186/1471-2148-11-78

Cite this article as: Cigliano et al:: Evolution of Parallel Spindles Like genes in plants and highlight of unique domain architecture ${ }^{\#}$. BMC Evolutionary Biology 2011 11:78.

\section{Submit your next manuscript to BioMed Central and take full advantage of:}

- Convenient online submission

- Thorough peer review

- No space constraints or color figure charges

- Immediate publication on acceptance

- Inclusion in PubMed, CAS, Scopus and Google Scholar

- Research which is freely available for redistribution

Submit your manuscript at www biomedcentral.com/submit
C Biomed Central 